\title{
Efeito de Um Mês de Destreinamento Sobre a Aptidão Física Relacionada à Saúde em Programa de Mudança de Estilo de Vida
}

\section{Effects of One Month Detraining Over Health-Related Physical Fitness in a Lifestyle Change Program}

\author{
Edilaine Michelin' \\ Christianne de Faria Coelho ${ }^{1,2}$ \\ Roberto Carlos Burini 1,2 \\ 1. Centro de Metabolismo em \\ Exercício e Nutrição (CeMENutri) \\ - Departamento de Saúde Pública \\ - Faculdade de Medicina de \\ Botucatu - Botucatu/SP \\ 2. Departamento de Alimentos \\ - FCF- PRONUT-USP - São Paulo/SP \\ Endereço para correspondência: \\ Rua Padre Salústio Rodrigues \\ Machado, 540 - Bairro: Vila dos \\ Lavradores - CEP 18.609-610 \\ - Botucatu - SP \\ Email: edimichelin@yahoo.com.br
}

Submetido em 27/02/2007 Versão final recebida em 17/10/2007 Aceito em 03/01/2008

\begin{abstract}
RESUMO
O exercício físico induz adaptações hemodinâmicas/ventilatórias e neuromusculares que podem ser revertidas com a interrupção do mesmo. O presente estudo teve por objetivo avaliar o efeito do destreinamento sobre a aptidão física relacionada à saúde. Foram avaliados 44 indivíduos de ambos os sexos com idade de 57,6 \pm 8,9 anos. O Protocolo de Mudança de Estilo de Vida Mexa-se Pró-Saúde com orientação nutricional e exercícios físicos supervisionados, teve duração de nove meses envolvendo exercícios aeróbicos, de resistência muscular localizada e flexibilidade, com duração de $80 \mathrm{~min} / \mathrm{sessão,} \mathrm{em} \mathrm{cinco} \mathrm{sessões}$ semanais. Foram selecionados os indivíduos que obtiveram freqüência mínima de $3 x /$ semana. 0 período de interrupção do treinamento foi de um mês. Foram avaliados peso $(\mathrm{kg})$ e estatura $(\mathrm{m})$ e calculado o Índice de Massa Corporal $\left(\mathrm{IMC}-\mathrm{kg} / \mathrm{m}^{2}\right)$ e realizados testes motores para flexibilidade (FLEX), força de membros inferiores (FMI) e superiores (FMS) e consumo máximo de oxigênio $\left(\mathrm{VO}_{2 \text { máx. }}\right)$ no início do programa (MI), após nove meses de treinamento (MT) e após pausa de um mês (MD). O tratamento estatístico utilizado foi ANOVA com nível de significância de 5\% e o teste de Tukey para a localização das diferenças entre os grupos, quando a mesma foi constatada. Os resultados mostraram que ganhos significativos de $22 \%$ e $7 \%$ na força de membros inferiores $\mathrm{eVO}_{2 \text { máx. }}$ respectivamente, alcançados com o treinamento foram mantidos após a interrupção do programa por um mês; enquanto que o aumento de $8 \%$ na flexibilidade retornou aos níveis basais após o período de destreinamento. Conclui-se que embora mantidos os ganhos de força de membros inferiores e capacidade aeróbica adquiridos, um mês de destreinamento foi suficiente para perder a flexibilidade conquistada.
\end{abstract}

Palavras-chave: exercício físico, saúde, interrupção do treinamento.

\section{ABSTRACT}

Physical exercise induces hemodynamic/ventilatory and neuromuscular adaptations that can be reverted with interruption of the training program. The aim of the present study was to evaluate the effect of detraining on physical fitness related to health. Forty-four healthy subjects, both male and female, aged $57.6 \pm 8.9$ years performed the 'Mexa-se Pró-Saúde' protocol with nutritional orientation and supervised physical exercises for nine months. The program consisted of aerobic, localized muscular endurance and flexibility exercises, with duration 80 minutes/session, five days/week. Only subjects who participated in the program for more than three days/week have been selected. The detraining period was one month. Weight $(\mathrm{kg})$ and height $(\mathrm{m})$ were measured and the body mass index (BMI) calculated. Additionally, motors tests to evaluate the flexibility (FLEX), strength of lower limbs (SLL) and upper limbs (SUL), and maximal oxygen uptake $\left(\mathrm{VO}_{2 \text { max }}\right)$ were conducted in the beginning of the study $(\mathrm{MI})$, after nine months of practicing (MT) and after detraining period (MD). ANOVA $(p<0.05)$ and Tukey test to show the difference between groups when it evidence were used for statistical treatment. The results showed that the gains of $22 \%$ and $7 \%$ on $S L L$ and $V_{2 m a x}$ respectively, obtained with the training, have not changed during the detraining period; however, the flexibility gain of $8 \%$ returned back to baseline after the detraining period. BMI and SUL did not change during the study. Although the lower limbs strength gains and maximal oxygen uptake obtained have been kept, one month of detraining was enough for losing the flexibility acquired.

Keywords: physical exercise, health, training interruption. 


\section{INTRODUÇÃO}

O sedentarismo, problema de saúde pública mundial, associado à outros fatores de risco modificáveis como tabagismo e alimentação inadequada são as principais causas das doenças crônicas não transmissíveis (DCNT)(1). Tais comportamentos associados às perdas fisiológicas inerentes à idade como modificações na composição corporal, força muscular, capacidade aeróbica e flexibilidade ${ }^{(2,3)}$, considerados componentes da aptidão física relacionados à saúde, agravam ainda mais o quadro das DCNT.

Por outro lado, o estilo de vida ativo e o bom condicionamento aeróbico estão associados, de forma independente, à redução da incidência de DCNT, de mortalidade geral e por doenças cardiovasculares( ${ }^{(4)}$. Além disso, o exercício físico pode aprimorar a realização das tarefas do cotidiano com aumento da força e resistência muscular ${ }^{(5,6)}$, aptidão aeróbia ${ }^{(7)}$, flexibilidade ${ }^{(3)}$ e evitar ou minimizar o aumento da gordura visceral e a sarcopenia que estão associadas à síndrome metabólica e incapacidade funcional(8-10).

Se por um lado a prática de exercícios físicos promove benefícios morfológicos, fisiológicos e funcionais, o tempo necessário para que essas melhoras sejam mantidas após a interrupção no treinamento permanece controverso ${ }^{(6,7,11,12)}$.

Essa interrupção, também conhecida como destreinamento, leva a perda parcial ou completa das adaptações anatômicas, fisiológicas e de desempenho induzidas pelo treinamento ${ }^{(13)}$ e variam quantitativa e qualitativamente dependendo do período de pausa.

Sendo assim, o objetivo do presente estudo foi avaliar o efeito de um mês de destreinamento sobre as melhoras de componentes da aptidão física relacionada à saúde conquistados por adultos participantes de um programa de mudança de estilo de vida (MEV).

\section{MÉTODOS}

\section{Sujeitos}

A amostra foi constituída por 44 indivíduos (19 homens e 25 muIheres) com idade entre 36 e 74 anos participantes do Programa de MEV. As características de aptidão física e de composição corporal dos indivíduos no início do estudo estão descritas na tabela 1.

O programa de MEV proposto no presente estudo, denominado "Mexa-se Pró-Saúde", foi criado e implantado pelo Centro de Metabolismo, Exercício e Nutrição (CeMENutri-UNESP-Botucatu) em 1991 e visa promover a melhora da saúde e qualidade de vida da população adulta e idosa de Botucatu mediante (re)educação alimentar e prática de exercícios físicos supervisionados. A capacidade funcional e o diagnóstico de DCNT são obtidos periodicamente por meio de avaliações

Tabela 1. Caracterização da amostra no início do estudo.

\begin{tabular}{lc}
\hline & MI (Média \pm DP) \\
\hline Idade (anos) & $57,6 \pm 8,8$ \\
Peso (kg) & $72,1 \pm 14,1$ \\
Estatura (m) & $1,63 \pm 0,08$ \\
IMC (kg/m $\left.{ }^{2}\right)$ & $26,9 \pm 4,1$ \\
Flex (cm) & $26,1 \pm 8,4$ \\
FMI (repetições) & $17,4 \pm 3,0$ \\
FMS (kg) & $34,6 \pm 10,5$ \\
VO & 2máx. \\
\hline
\end{tabular}

médica, nutricional, laboratorial e do desempenho físico e realizado por profissionais e estudantes de Educação Física, Nutrição, Bioquímica, Biologia, Biomedicina e Medicina.

Os voluntários assinaram termo de consentimento livre e esclarecido informando-os sobre a proposta e os procedimentos do estudo de acordo com os preceitos éticos da Resolução no 196 de 10/outubro/1996. O projeto recebeu parecer favorável (OF 107/2004-CEP) do Comitê de Ética em Pesquisa da Faculdade de Medicina de Botucatu em 05 de abril de 2004.

Foram selecionados para o estudo apenas os indivíduos que obtiveram freqüência mínima de três vezes/semana ao protocolo de exercícios físicos e que compareceram aos três momentos de avaliação. Foram excluídos aqueles que possuíssem complicações cardíacas, limitações ortopédicas ou outra patologia considerada incapacitante detectada no início do estudo por meio da triagem médica.

\section{Avaliação da Composição Corporal}

Foram avaliados peso corporal através de balança antropométrica (Filizola ${ }^{\circledR}$, Brasil), com precisão de 0,1 kg, estatura por meio de estadiômetro fixo com precisão de 0,1 cm e calculado o índice de massa corpórea (IMC) por meio do quociente peso corporal/estatura ${ }^{(2)}\left(\mathrm{kg} / \mathrm{m}^{2}\right)$. A classificação para IMC foi realizada de acordo com a OMS ${ }^{(14)}$.

\section{Avaliação do desempenho motor}

A flexibilidade de tronco (FLEX) foi avaliada através do teste de sentar e alcançar utilizando um banco de madeira de $48 \mathrm{~cm}$ com uma fita métrica de $55 \mathrm{~cm}$ fixada ao mesmo, iniciando em zero na parte mais próxima ao indivíduo. Foram realizadas três tentativas sendo aceito o maior valor ${ }^{(15)}$. Para avaliar a força muscular de membros inferiores (FMI) foi utilizado o teste de sentar e levantar da cadeira e computado o maior número de repetições no período de 30 segundos $^{(16,17)}$. A força muscular de membros superiores (FMS) foi determinada através do teste de preensão manual com dinamômetro hidráulico com escala de 0 a $100 \mathrm{~kg}$. Para avaliação do consumo máximo de oxigênio $\left(\mathrm{VO}_{2 \mathrm{máx}}\right)$ utilizou-se o teste indireto de caminhada da milha em pista de 400 metros $^{(18)}$. No final do percurso anotaram-se o tempo para percorrer uma milha (1609 m) e a freqüência cardíaca ( $F C$ ) monitorado através de freqüencímetro Polar ${ }^{\circledR} \mathrm{A} 1^{\mathrm{TM}}$. O V $\mathrm{VO}_{2 \text { máx }}$ foi calculado utilizando a seguinte fórmula: $132,853-(0,1692 *$ peso corporal em $\mathrm{kg})-(0,3877 *$ idade em anos $)+(6,315 *$ sexo $)-(3,2649 *$ tempo em minutos $)-(0,1565 * \mathrm{FC})$, sendo adotado o valor 0 para mulher e 1 para homem e a freqüência cardíaca $(F C)$ ao final da caminhada de uma milha ${ }^{(19)}$.

\section{Protocolo de exercícios físicos}

O protocolo de exercícios físicos foi conduzido por profissionais de Educação Física, oferecido em cinco sessões semanais com duração de 80 minutos/sessão, durante nove meses e dividia-se em: Programa A ( $2^{\text {as }}, 4^{\text {as }}$ e $6^{\text {as }}$ feiras) - alongamento muscular (10 minutos), aquecimento com danças (10 minutos), caminhada (40 minutos - 65 a $85 \%$ da $F C_{\text {máx. }}$ predita pela fórmula 220-idade) e flexibilidade (20 minutos - exercícios de alongamento muscular) e Programa B - realizado às $3^{\text {as }}$ e $5^{\text {as }}$ feiras - alongamento muscular (5 minutos), aquecimento com danças (5 minutos), resistência muscular localizada (30 minutos - exercícios com pesos livres para peitorais, dorsais, deltóides, tríceps, bíceps, extensores, flexores, adutores e abdutores da coxa e abdominais em 3 séries de 10 a 15 repetições), caminhada (30 minutos - 65 a 85\% da $\mathrm{FC}_{\text {Max. }}$. predita pela fórmula 220-idade) e relaxamento (10 minutos - volta à calma ou resfriamento com alongamento muscular leve). Os equipamentos utilizados foram halteres, 
caneleiras, bolas de borracha, bastões de madeira e colchonetes. 0 período de destreinamento foi de um mês e coincidia com o período de férias dos participantes.

\section{Análise Estatística}

Foram calculados os valores de média, desvio padrão e intervalos de confiança de $95 \%$ para as variáveis da composição corporal e da aptidão física ao longo dos três momentos de avaliação MI (inicial), MT (nove meses) e MD (pausa de um mês). A diferença entre os momentos (delta) e entre as médias foram analisadas pela ANOVA/MANOVA e para localizá-las empregou o teste de "post-hoc" de Tukey, adotando-se nível de significância de 5\%.

\section{RESULTADOS}

Na tabela 2 são descritos os resultados da composição corporal (peso e IMC) e dos testes motores para flexibilidade, força de membros superiores e inferiores e capacidade aeróbica no início do programa (MI), após nove meses de exercícios (MT) e um mês após a interrupção do mesmo (MD).

No momento inicial verifica-se que, em média, os indivíduos encontravam-se na classificação de sobrepeso quanto ao IMC. O programa de exercícios bem como o destreinamento não exerceram efeitos significativos sobre o peso corporal e IMC, sendo que os mesmos permaneceram inalterados tanto em valores como em sua classificação.

A flexibilidade apresentou aumento significativo de $8 \%$ após o período de treinamento, porém um mês de interrupção causou redução, também significativa, nesse componente, retornando os valores próximos aos basais.

$\mathrm{Na}$ força de membros inferiores (FMI), o treinamento exerceu efeito positivo aumentando-a significativamente em 22\%, já a interrupção de um mês não a influenciou, mantendo seus valores próximos àqueles do período de treinamento. Ao contrário da FMI, a força de membros superiores (FMS) não apresentou melhora com o treinamento e tampouco se alterou com um mês de destreinamento.

Para a capacidade aeróbica, observou-se variações significativas nos valores de $\mathrm{VO}_{2 \text { máx }}$ com melhora de $7 \%$ após nove meses de exercícios e tendência à queda após interrupção no treinamento, porém, sem diferenciação estatística.

\section{DISCUSSÃO}

O presente estudo objetivou avaliar o efeito de um mês de destreinamento sobre a aptidão física relacionada à saúde e os resultados indicam que a maioria dos benefícios alcançados com um programa de exercícios físicos não se perderam com a interrupção do mesmo pelo período de um mês.
O protocolo de exercícios foi oferecido em cinco sessões semanais, com duração de 80 minutos/sessão e foram selecionados apenas os indivíduos que obtiveram freqüência mínima de 3x/semana que corresponderia a 240 minutos/semana de atividade física satisfazendo assim as recomendações da $\mathrm{OMS}^{(1)}$ e do Colégio Americano de Medicina Esportiva $\left(\right.$ ACSM) ${ }^{(18)}$.

De acordo com informações disponíveis na literatura, a forma mais aceita de melhorar consideravelmente a aptidão física é através da realização de exercícios físicos. No entanto, existe uma notória controvérsia com relação ao tempo de pausa necessário para que os efeitos produzidos pelo exercício na aptidão sejam revertidos ${ }^{(6,7,11,12)}$.

Os resultados desse trabalho mostram que peso corporal e IMC não alteraram significativamente durante o período estudado, dados esses que corroboram com o de outro estudo onde não foram observadas alterações significativas nesses indicadores da composição corporal tanto com o treinamento como com o destreinamento, ambos de oito semanas ${ }^{(6)}$.

Esses achados suportam a afirmação do ACSM apontando que a composição corporal pode modificar apenas discretamente após várias semanas de treinamento físico sem restrição dietética e as recomendações atuais para controle da obesidade apóiam o uso de durações maiores de atividade física (200 a 300 minutos diários)(20), o que pode ter influenciado nos resultados, pois a duração das sessões de exercício foi menor do que a preconizada e provavelmente insuficiente para modificar a composição corporal dos indivíduos.

Quanto ao efeito do destreinamento, assim como no estudo citado anteriormente ${ }^{(6)}$, o período de pausa pode ter sido curto para promover qualquer mudança no peso corporal e no IMC dos indivíduos, uma vez que reduções foram observadas apenas após 12 semanas de paralisação(21) e, levando em consideração que não verificou-se alteração com o treinamento, tampouco seria provável verificarmos qualquer mudança com o destreinamento.

Quando expresso em valores médios o IMC não alterou, porém, quando classificamos individualmente a amostra notamos que após nove meses de exercício, cinco indivíduos foram beneficiados, sendo que três deles classificados como sobrepeso se reclassificaram para eutróficos, um obeso grau I reclassificou-se para sobrepeso e um indivíduo obeso grau II reclassificou-se para obeso grau I. Da mesma forma, observamos que o período de destreino foi deletério para seis indivíduos sendo que cinco deles se reclassificaram de eutróficos para sobrepeso e um de obeso grau II para obeso grau III.

Com relação ao desempenho motor, a literatura cita maior perda na massa muscular dos 40 aos 69 anos de idade(8) e redução nas fibras musculares do tipo II com conseqüente redução da força muscular(22)

Tabela 2. Média de Peso, Índice de Massa Corporal (IMC), flexibilidade (FLEX), força de membros inferiores (FMI) e superiores (FMS) e capacidade aeróbica (VO ${ }_{2 m a ́ x}$ ), respectivos resultados do teste estatístico e intervalos de confiança de 95\%, nos diferentes momentos de avaliação.

\begin{tabular}{|c|c|c|c|c|}
\hline & MI (Inicial) & MT (9 meses) & MD (pausa 1mês) & $\Delta \%$ (MI-MT/MT-MD) \\
\hline PESO (kg) & $72,1 \pm 14,1^{\mathrm{a}}(67,8-76,3)$ & $71,5 \pm 13,7^{\mathrm{a}}(67,3-75,6)$ & $72,2 \pm 13,7^{\mathrm{a}}(68-76,4)$ & $-0,8 /+0,9$ \\
\hline IMC $\left(\mathbf{k g} / \mathrm{m}^{2}\right)$ & $26,9 \pm 4,1^{\mathrm{a}}(25,7-28,1)$ & $26,7 \pm 3,8^{\mathrm{a}}(25,5-27,9)$ & $26,9 \pm 3,9^{\mathrm{a}}(25,7-28,1)$ & $0,7 /+0,9$ \\
\hline FLEX (cm) & $26,1 \pm 8,3^{\mathrm{a}}(23,6-28,6)$ & $28,1 \pm 7,6^{b}(25,8-30,4)$ & $26,5 \pm 7,6^{a}(24,2-28,8)$ & $+8 /-6$ \\
\hline FMI (repetições) & $17,4 \pm 3,0^{\mathrm{a}}(16,5-18,3)$ & $21,3 \pm 3,1^{b}(20,4-22,2)$ & $21,8 \pm 3,5^{b}(20,7-22,9)$ & $+22 /+2$ \\
\hline FMS (kg) & $34,6 \pm 10,5^{a}(31,4-37,8)$ & $33,1 \pm 10,8^{\mathrm{a}}(29,8-36,4)$ & $34,6 \pm 10,8^{a}(31,3-37,9)$ & $-4 /+4$ \\
\hline $\mathrm{VO}_{2 \text { máx. }}(\mathrm{ml} / \mathrm{kg} / \mathrm{min})$ & $32,9 \pm 7,7^{a}(30,6-35,2)$ & $35,1 \pm 8,3^{b}(32,6-37,6)$ & $34,0 \pm 8,2^{2 \mathrm{~b}}(31,5-36,5)$ & $+7 /-3$ \\
\hline
\end{tabular}

Médias seguidas de mesma letra não diferem significativamente ao nível de 5\% pelo teste de Tukey. 
O presente estudo apresentou na avaliação inicial para FMI, cinco indivíduos classificados abaixo da média e um na média. Após a intervenção observou-se aumento significativo de $22 \%$ nesse componente, sendo que os mesmos seis indivíduos reclassificaram para a condição acima da média, igualando-se aos demais. Com o período de um mês de destreinamento a FMI não se alterou.

Os resultados obtidos com o treinamento são similares a um estudo em indivíduos com doença arterial coronariana (DAC) com aumento da FMI, avaliado pelo teste de 1RM, após quatro e oito meses de exercícios, porém, diferem quanto às perdas induzidas pela pausa no exercício, pois estas foram significativas após 12 semanas de inatividade ${ }^{(21)}$.

De maneira semelhante, observou-se incrementos de 34\% e 28\% na força de 1RM de extensão unilateral de joelho em jovens e idosos, respectivamente, após treinamento resistido de nove semanas. As perdas foram observadas apenas após 31 semanas de interrupção dos exercícios e foram de $14 \%$ nos idosos e $8 \%$ nos jovens ${ }^{(23)}$, comprovando que o destreino reduz a força independente da idade.

No entanto, após 12 semanas de treinamento resistido aplicado em oito mulheres saudáveis ( 56 a 81 anos), verificou-se melhora significativa para FMI (teste de $1 \mathrm{RM}$ para agachamento e leg press $45^{\circ}$ ), porém, quatro semanas de destreinamento somente afetou significativamente o teste no leg press $45^{\circ}$, enquanto o exercício agachamento apresentou queda, porém não significativa ${ }^{(11)}$.

Sugere-se que a FMI obteve benefícios significativos com o treinamento adotado no presente estudo devido ao mesmo contemplar atividades de caminhada e resistência muscular localizada (RML) e que o período de paralisação do programa (um mês) seja insuficiente para alterar esse componente, uma vez que os estudos comparativos utilizaram períodos maiores de destreinamento.

A força de membros superiores não sofreu influência do programa de exercícios nem do período de destreino. Em avaliação inicial constatou-se que $75 \%$ da amostra apresentava classificação média e boa para FMS, condição que se manteve nos outros dois momentos de avaliação e que pode ser explicada pela maior utilização desta musculatura nas atividades do cotidiano (lavar e passar roupas, carregar baldes e crianças, cuidar do jardim ou quintal, capinar, etc).

Comportamento parecido foi observado quando avaliaram, pelo teste de preensão manual, os efeitos de um programa de exercícios aeróbicos em 23 mulheres de 50 a 59 anos de idade e não encontraram melhora significativa na força de membros superiores ${ }^{(3)}$.

Com a utilização do teste de 1RM para determinar a FMS, notouse significativa melhora nesse componente em pacientes com DAC após oito meses de exercícios aeróbicos e resistidos e a interrupção do treinamento por três meses reduziu-a significativamente ${ }^{(21)}$. Quando empregado o teste de 10RM como método de avaliação, mulheres pós-menopausadas obtiveram resultados positivos com treinamento de força e resistência muscular e a interrupção de oito semanas reverteu os resultados, levando à diminuição da FMS(6).

Sugere-se que a divergência de resultados seja explicada pelo método de avaliação da força, uma vez que os estudos que apresentaram reduções com o destreinamento fizeram uso de testes para grupamentos musculares maiores e no presente estudo a avaliação foi feita em um grupamento muscular considerado menor. Outra hipótese é que o teste pode não ser suficientemente sensível para detectar mudanças, ou ainda, o período de pausa foi insuficiente para causar alterações nesse componente.
Além disso, torna-se difícil avaliar perdas em função da interrupção do exercício em componente que não obteve ganho com o mesmo, principalmente numa amostra com boa classificação inicial.

Quanto à capacidade aeróbica, estudos mostram declínio aproximado de 2,9\% e 1,5\% ao ano, no $\mathrm{VO}_{2 \text { máx. }}$ de atletas e sedentários, respectivamente( ${ }^{(24)}$, e idosos exibindo valores de $\mathrm{VO}_{2 \text { máx. }}$ menores quando comparados com jovens ${ }^{(23)}$, comprovando que idade, gênero, nível de atividade física, hereditariedade, aumento na gordura corporal e redução na massa de músculo esquelético têm importante influência nesse componente ${ }^{(2)}$.

Entretanto, a participação em atividades aeróbicas regulares pode elevar o consumo de oxigênio em 10\% a 25\%(25). No presente estudo observamos melhora de $7 \%$ nos valores de $\mathrm{VO}_{2 \text { máx }}$ após nove meses de exercício, sendo que cinco indivíduos foram reclassificados de regular para bom, sete de bom para ótimo, um de fraco para bom, um de fraco para regular e um de fraco para ótimo, quando comparado à avaliação inicial. Isto pode ter ocorrido em função do aumento obtido na FMl que contribuiu para melhorar o tempo no teste da milha.

Após um mês de destreinamento houve tendência à queda nos valores de $\mathrm{VO}_{2 \text { máx, }}$ resultados confirmados por estudo utilizando protocolo de exercícios resistidos e aeróbios em pacientes com DAC, o qual verificou redução significativa apenas após três meses de destreinamento, pós ganho de 15\% durante oito meses de exercícios ${ }^{(21)}$.

Analisando os resultados em outras populações e faixas etárias, observamos que o efeito de oito semanas de treinamento aeróbico em mulheres sedentárias desaparece após quatro a doze semanas de inatividade ${ }^{(26)}$, bem como em idosos após 16 semanas de exercícios de resistência e quatro semanas de destreinamento(7), dados esses respaldados pela literatura que cita períodos superiores a duas semanas de inatividade como responsáveis por perdas na aptidão cardiorrespiratória(27).

Embora não significativo, o declínio dos valores de $\mathrm{VO}_{2 \text { máx }}$ observado após o período de destreinamento pode ser considerado um resultado relevante do ponto de vista biológico pois nova reclassificação ocorreu após pausa de um mês, sendo que dez indivíduos foram transferidos para níveis inferiores de condicionamento.

Alterações no componente flexibilidade afetam a qualidade de vida dos indivíduos, pois interferem na mobilidade e capacidade funcional ${ }^{28}$ que, por sua vez, prejudicam a realização das AVDs. Por outro lado, exercícios que aumentam a flexibilidade contribuem para a redução das injúrias músculo-esqueléticas, dores articulares e quedas, esta última principalmente entre indivíduos idosos.

No presente estudo, o protocolo de exercício melhorou a flexibilidade dos indivíduos estudados, sendo que 17 sujeitos melhoraram a classificação após nove meses de exercício, embora a condição de sobrepeso dos mesmos tenha se mantido durante o estudo, ou seja, não houve mudanças na composição corporal o que poderia influenciar na realização do teste proposto. Após um mês de destreinamento, 14 indivíduos tiveram sua FLEX reduzida, mostrando que a interrupção do treinamento teve efeito deletério neste componente.

Em estudo longitudinal com mulheres na faixa etária de 50-59 anos, realizando protocolo de exercícios aeróbicos, de alongamento e flexibilidade por seis meses, notou-se discreto aumento de 15\% no componente FLEX, porém sem diferença estatística quando comparado aos valores iniciais ${ }^{(3)}$.

Um programa de treinamento multicomponente (exercícios aeróbicos, de resistência muscular e flexibilidade) melhorou significativamente a flexibilidade de 12 jovens idosos, após nove semanas de exercícios 
e com uma pausa de duas semanas iniciou-se o processo de perda nesse componente, sendo que para o período de destreinamento de seis semanas, a redução já era significativa em relação ao período de treinamento ${ }^{(29)}$, porém, o método de avaliação e a faixa etária estudada foi diferente do nosso estudo o que pode ter determinado a diferença nos resultados.

Encontramos estudos avaliando o efeitos do exercício e do destreinamento sobre a flexibilidade, mas sempre em populações mais idosas e supomos que esse componente influencie mais a aptidão física dessa população, por isso a escassez de trabalhos com jovens e indivíduos de meia idade, fato esse que dificultou a discussão dos resultados nesse componente.

Considerando que o aumento do peso corporal pode influenciar o desempenho motor, no presente estudo a manutenção da composição corporal nos permite descartar a influência dessa variável sobre os testes motores dos indivíduos durante todo o período experimental.

Faz-se importante salientar que o estudo apresenta limitações como a falta de intervenção nutricional (alterações na composição da dieta), que poderia influenciar a composição corporal, uma vez que foram realizadas apenas avaliações da ingestão alimentar e orientação nutricional por meio de palestras mensais coletivas incentivando mudanças alimentares e, divisão dos indivíduos por gênero, já que diferenças fisiológicas entre os sexos são bem estabelecidas.

\section{CONCLUSÃO}

Dos componentes que melhoraram significativamente após nove meses de intervenção com programa de exercícios físicos (força de membros inferiores, aptidão cardiorespiratória $\mathrm{VO}_{2 \text { máx. }}$ e flexibilidade), o período de um mês de destreinamento exerceu efeito deletério apenas sobre a flexibilidade. A força muscular de membros superiores e composição corporal não alteraram com a intervenção nem tampouco com o período de interrupção do treinamento.

Todos os autores declararam não haver qualquer potencial conflito de interesses referente a este artigo.

\section{REFERÊNCIAS BIBLIOGRÁFICAS}

1. World Health Organization - WHO. Draft global strategy on diet, physical activity and health - integrated prevention of noncommunicable diseases. Geneva, 2003; 3-18 (WHO Technical Report Series).

2. Fleg $J$, Morrell $C H$, Bos $A G$, Brant $L$, Talbot $L A$, Wright JG, et al. Accelerated longitudinal decline of aerobic capacity in healthy older adults. Circulation. 2005; 112: 674-82

3. Matsudo SM, Matsudo VKR, Barros Neto TL, Araújo TL. Evolução do perfil neuromotor e capacidade funcional de mulheres fisicamente ativas de acordo com a idade cronológica. Rev Bras Med Esporte. 2003; 9: 1-12.

4. Barreto SM, Pinheiro ARO, Sichieri R, Monteiro CA, Filho MB, Schimidt MI, et al. Análise da estratégia global para alimentação, atividade física e saúde, da Organização Mundial da Saúde. Epidemiol Serv Saúde. 2005; 14: 41-68.

5. Roth SM, Ivey FM, Martel GF, Lemmer JT, Hurlbut DE, Siegel EL, et al. Muscle size responses to strenght training in young and older men and women. J Am Geriatr Soc. 2001; 49: 1428-33.

6. Elliott KJ, Sale C, Cable NT. Effects of resistance training and detraining on muscle strength and blood lipid profiles in postmenopausal women. Br J Sports Med. 2002; 36: 340-5.

7. Fatouros JG, Jamurtas AZ, Villiotou V, Pouliopoulou S, Fotinakis P, Taxildaris K, et al. Oxidative stress responses in older men during endurance training and detraining. Med Sci Sports Exerc. 2004; 36: 2065-72.

8. Janssen I, Heymsfield SB, Ross R. Low relative skeletal muscle mass (sarcopenia) in older persons is associated with functional impairment and physical disability. JAGS. 2002; 50: 889-96.

9. Lakka TA, Laaksonen DE, Lakks HM, Nnikko NMA, Niskanen LK, Rauramaa R, et al. Sedentary lifestyle, poor cardiorespiratory fitness, and the metabolic syndrome. Med Sci Sports Exerc. 2003; 35: 1279-86.

10. Jurca R, Lamonte MJ, Church TS, Earnest CP, Fitzgerald SJ, Barlow CE, et al. Associations of muscle strength and aerobic fitness with metabolic syndrome in men. Med Sci Sports Exerc. 2004; 36: 1301-7.

11. Raso V, Matsudo SMM, Matsudo VKR. A força de mulheres idosas decresce principalmente após oito semanas de interrupção de um programa de exercícios com pesos livres. Rev Bras Med Esporte. 2001; 7: 177-86.

12. Salmela LFT, Santiago L, Lima RCM, Lana DM, Camargos FFO, Cassiano JG. Functional perfomance and quality of life related to training and detraining of community-dwelling elderly. Disabil Rehabil. 2005; $27: 1007-12$.

13. Mujika I, Padilla S. Detraining: loss of training-induced physiological and performance adaptations. Part I - short term insufficient training stimulus. Sports Med. 2000; 30: 79-87.

14. World Health Organization - WHO. Diet, nutrition and the prevention of chronic diseases. Geneva 2002: 68-9.
15. Nahas MV. Aptidão musculoesquelética: força e mobilidade. In: Atividade física, saúde e qualidade de vida: conceitos e sugestões para um estilo de vida ativo. Londrina: Midiograf; 2001: 57-78.

16. Jones CJ, Rikli RE, Beam WC. A 30-s chair-stand test as a measure of lower body strenght in community-residing older adults. Res Q Exerc Sport. 1999; 70: 113-9.

17. Rikli RE, Jones CJ. Development and validation of a functional fitness test for community-residing older adults. J Aging Phys Act. 1999; 7: 129-61.

18. American College of Sports Medicine. ACSM's guidelines for exercise testing and prescription. 6 ed. Filadélfia: Lippincott, 2001:47-8.

19. Kline $G \mathrm{M}$, Porcari JP, Hintermeister R. Estimation of $\mathrm{VO}_{2 \text { máx }}$ from a one-mile track walk, gender, age and body weight. Med Sci Sports Exerc. 1987; 19: 253-9.

20. American College of Sports Medicine. Position Stand. Appropriate intervention strategies for weight loss and prevention of weight regain for adults. Med Sci Sports Exerc. 2001; 33: 2145-56.

21. Tokmakidis SP, Volaklis KA. Training and detraining effects of a combined-strength and aerobic exercise program on blood lipids in patients with coronary artery disease. J Cardiopulm Rehabil. 2003; 23: 193-200

22. Lee WS, Cheung WH, Qin L, Tang N, Leung KS. Age-associated Decrease of Type IIA/B Human Skeletal Muscle Fibers. Clin Orthop Relat Res. 2006; 450: 231-7.

23. Lemmer JT, Hurbut DE, Martel GF, Tracy BL, Ivey FM, Metter EJ, et al. Age and gender responses to strength training and detraining. Med Sci Sports Exerc. 2000; 32: 1505-12.

24. Katzel $\mathrm{LI}$, Sorkin JD, Fleg JL. A comparison of longitudinal changes in aerobic fitness in older endurance athletes and sedentary men. J Am Geriatr Soc. 2001; 49: 1657-64.

25. Spirduso WW. Physical dimensions of aging. Champaign, LL: Human Kinetics; 1995: 432.

26. Wang JS, Jen $\mathrm{CJ}$, Chen HI. Effects of chronic exercise and deconditioning on platelet function in women. J Appl Physiol. 1997; 83: 2080-5.

27. Mujika I, Padilla S. Cardiorespiratory and metabolic characteristics of detraining in humans. Med Sci Sports Exerc. 2001; 33: 413-21.

28. Janssen I, Heymsfield SB, Baumgartner RN, Ross R. Estimation of skeletal muscle mass by bioelectrical impedance analysis. J Appl Physiol. 2000; 89: 465-71.

29. Toraman NF, Ayceman N. Effects of six weeks of detraining on retention of functional fitness of old people after nine weeks of multicomponent training. Br J Sports Med. 2005; 39: 565-8. 